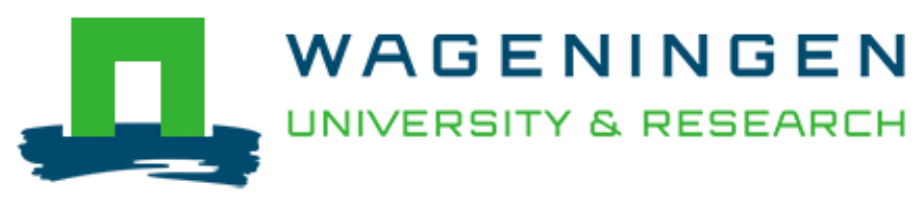

Sign Switch of Gaussian Bending Modulus for Microemulsions: A SelfConsistent Field Analysis Exploring Scale Invariant Curvature Energies

\author{
Varadharajan, R., \& Leermakers, F. A. M.
}

This is a "Post-Print" accepted manuscript, which has been published in "Physical Review Letters"

This version is distributed under a non-commercial no derivatives Creative Commons (c) (1) () $\Theta$ (CC-BY-NC-ND) user license, which permits use, distribution, and reproduction in any medium, provided the original work is properly cited and not used for commercial purposes. Further, the restriction applies that if you remix, transform, or build upon the material, you may not distribute the modified material.

Please cite this publication as follows:

Varadharajan, R., \& Leermakers, F. A. M. (2018). Sign Switch of Gaussian Bending Modulus for Microemulsions: A Self-Consistent Field Analysis Exploring Scale Invariant Curvature Energies. Physical Review Letters, 120(2), [028003]. DOI: 10.1103/PhysRevLett. 120.028003

You can download the published version at:

https://doi.org/10.1103/PhysRevLett.120.028003 


\title{
Sign switch of Gaussian bending modulus for microemulsions; a self-consistent field analysis exploring scale invariant curvature energies
}

\author{
R. Varadharajan* and F. A. M. Leermakers ${ }^{\dagger}$ \\ Physical Chemistry \& Soft Matter, Wageningen University and Research Center, \\ Stippeneng 4, 6708 WE Wageningen, The Netherlands.
}

(Dated: October 16, 2017)

\begin{abstract}
Bending rigidities of tensionless balanced liquid-liquid interfaces as occurring in microemulsions are predicted using self-consistent field theory for molecularly inhomogeneous systems. Considering geometries with scale invariant curvature energies gives unambiguous bending rigidities for systems with fixed chemical potentials: The minimal surface Im3m cubic phase is used to find the Gaussian bending rigidity, $\bar{\kappa}$, and a torus with Willmore energy $W=2 \pi^{2}$ allows for direct evaluation of the mean bending modulus, $\kappa$. Consistent with this, the spherical droplet gives access to $2 \kappa+\bar{\kappa}$. We observe that $\bar{\kappa}$ tends to be negative for strong segregation and positive for weak segregation; a finding which is instrumental for understanding phase transitions from a lamellar to a sponge-like microemulsion. Invariably, $\kappa$ remains positive and increases with increasing strength of segregation.
\end{abstract}

Interfaces characterized by dense surfactant packings, such as microemulsions [1] and biological membranes [2] that are found naturally or manipulated artificially to be in a state of near-zero tension, have extensive areas. Often such interfaces feature a spontaneous curvature that manifests in spherical or cylindrical (swollen) micelles $[3,4]$. When a system is tensionless and precisely balanced-typical for single component bilayers and expected for the middle-phase microemulsions - the interface's spontaneous curvature vanishes [5] and ultra-low interfacial energies can be achieved [6, 7]. Here, the elastic moduli, mean $(\kappa)$ and Gaussian $(\bar{\kappa})$ bending rigidities, control the interface fluctuations and topology, respectively. Such systems show a first-order phase transition from lamellar to sponge-like phases, e.g., upon an increase of the temperature for nonionic systems, and a change of the salinity for ionic systems $[6,8]$. A preeminent challenge is to predict, from a molecular model for such interfaces, a means to induce a sign change in the $\bar{\kappa}$ from negative to positive; this signals the loss of stability of the lamellar, $L_{\alpha}$, oil-surfactant-water ordering in favor of a phase with saddles, $L_{3}$ or sponge-like. Another long-standing problem is understanding the relation between surfactant chain architecture and corresponding bending rigidities $[9,10]$.

Earlier theoretical methods [11, 12], experiments [13$15]$, and simulations $[16,17]$ that attempted to link bending rigidities to molecular properties did not provide information on $\bar{\kappa}$; moreover, the results for $\kappa$ were not consistent with each other. Therefore uncertainties prevail and these persist also because internal checks for presented rigidities are rarely provided. As a result, there exists no accepted molecular level theory that convincingly links molecular characteristics to both mechanical parameters of the interfaces $(\kappa$ and $\bar{\kappa})$. Notably, the missing information for $\bar{\kappa}$ is remarkable as its magnitude and, in particular, its sign are fundamental to the understanding of microemulsions.

The primary obstacle in establishing a molecular model for determining bending rigidities is the requirement of curving the interface at fixed chemical potentials. In this letter, we propose an elegant protocol with internal checks to find these rigidities. We consider interfaces with scale invariant curvature energies and illustrate the protocol for tensionless, balanced liquid-liquid (L/L) interfaces. In line with experimental findings, we report the existence of a sign switch for $\bar{\kappa}$ which triggers a phase transition from planar to sponge-like phases in middlephase microemulsions. We focus on the role of the interaction parameter which in strong segregation has a large value and for weak segregation a small value; further, we elaborate on the role of the molecular weights of the solvents and that of the amphiphile.

Experiments, simulations, and calculations [11-17] reviewed above have major disadvantages and ambiguities because the systems featured too many complications. We examine a tensionless balanced interface which still is highly relevant to middle-phase microemulsion systems wherein oil and water are separated by a surfactant film with extensive areas and often a complex interface topology. Our focus on tensionless (interfacial tension $\gamma=0$ ) balanced (spontaneous curvature $J_{0}=0$ ) L/L interface avoids the complications of a finite Laplace pressure (i.e. $\left.\Delta P_{L}=0\right)$ when imposing some interfacial curvature. Such a model is readily implemented in the ScheutjensFleer Self-Consistent Field theory (SF-SCF) for molecularly inhomogeneous systems. We can consider this idealized system in three different geometries with scaleinvariant curvature energies. The latter is essential, as it allows for an analysis in the grand canonical ensemble $(\mu, V, T)$, which opens a convincing route to estimate the rigidities: (i) A spherically curved droplet with $\Delta P_{L}=0$ is used to find $2 \kappa+\bar{\kappa}$; (ii) A minimal $\operatorname{Im} 3 \mathrm{~m}$ surface (by construction has $\Delta P_{L}=0$ ) is used to find $\bar{\kappa}$; (iii) A minimal torus interface is used to find $\kappa$ also for conditions that $\Delta P_{L}=0$.

We note that the route to obtain rigidities of balanced tensionless L/L interfaces shows similarities but also im- 
portant differences from the symmetric freely dispersed lipid bilayers [18]. For bilayers, we could use the $\operatorname{Im} 3 \mathrm{~m}$ cubic phase and the spherical vesicle to find $\bar{\kappa}$ and $2 \kappa+\bar{\kappa}$, respectively. The cylindrically curved vesicle could be used to obtain $\kappa$ in two ways: (i) As the number of lipids per unit area is found to be a constant (i.e., not a function of the radius $R$ of the cylindrical vesicle), $\kappa$ was found from the excess Helmholtz energy per unit length, $F_{c}^{\sigma}$, i.e., $\kappa=R F_{c}^{\sigma} / \pi$; (ii) Realizing that the grand potential of the cylindrical vesicle per unit length $\Omega_{c}$ is split up equally into bending energy and stretching energy, $\kappa$ is also found from (half) the grand potential density per unit length, i.e. $\kappa=R \Omega_{c} /(2 \pi)$. However, for the tensionless balanced $\mathrm{L} / \mathrm{L}$ interface, curved in cylindrical geometry with $\Delta P_{L}=0, \kappa$ can neither be computed from the Helmholtz energy per unit length, nor from the grand potential per unit length, as there is neither a conservation of the number of surfactant per unit area nor a conservation of the chemical potentials of the molecules of the system, cf. Figs. (1c) and (1d) shown below. Importantly, in the L/L interface, we do not find a coincidental equal splitting of curvature and tension energies.

Following Helfrich, by expanding the interfacial tension $(\gamma)$ in mean curvature $\left(J=1 / R_{1}+1 / R_{2}\right)$ and Gaussian curvature $\left(K=1 / R_{1} R_{2}\right)$, with $R_{1}, R_{2}$ being principle radii of curvature as

$$
\gamma(J, K)-\gamma(0,0)=-\kappa J_{0} J+\frac{1}{2} \kappa J^{2}+\bar{\kappa} K,
$$

we identify $\gamma(J, K)$ as the appropriate characteristic function that carries the bending information for curving the interface at constant chemical potentials [19]. This expansion is the starting point for our analysis of interfacial equilibrium properties, as it appears, refer Fig. (1d), for the balanced L/L interface, we find curved interfaces that exist at chemical potentials equal to that of the ground state (tensionless balanced planar interfaces) not only for the surfactant but also for the two solvents. Note, that in this system, both $\gamma(0,0)=0$ and $J_{0}=0$ and Eqn. (1) simplifies to $\gamma(J, K)=\frac{1}{2} \kappa J^{2}+\bar{\kappa} K$.

Within SF-SCF framework, extremizing the mean field free energy for a molecularly inhomogeneous system provides both structural and accurate thermodynamic information [18, 20-22]. We have implemented a coarsegrained molecular model in which there are two types of spherically symmetric segments A and B. These segments are used in two solvents, each with length $n, A_{n}$ and $B_{n}$ forming the two liquid phases $\alpha$ and $\beta$, respectively, and in a diblock copolymer composed of blocks of equal length $N, A_{N} B_{N}$. This approach requires molecular partition functions, which are evaluated within a lattice considering the molecules as freely jointed chains. Accordingly, segments fit on lattice sites. The lattice sites are organized as homogeneously curved- or planar layers. Driven by the segregation between the segments, an interface develops on which the lattice geometry im-
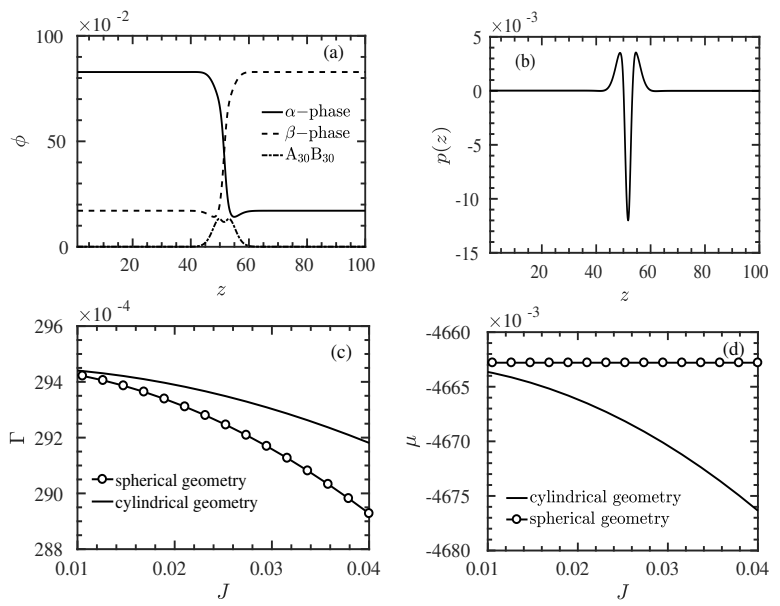

FIG. 1. (a) Volume fraction distribution and (b) Lateral pressure distribution (in $k_{B} T / b^{3}$ ) in a planar tensionless interface. (c) Area per surfactant molecule $(\Gamma)$ [in units $b^{2}$ ] and $(d)$ Chemical potential of surfactant $\left(\mu \equiv \mu_{s}\right)$ [in units of $k_{B} T$ ] as a function of interface curvature as indicated for systems with $\Delta P_{L}=0$. Surfactant $\mathrm{A}_{30} \mathrm{~B}_{30}$, solvents, $\mathrm{A}_{4}$ and $\mathrm{B}_{4} \cdot \chi=0.6$

poses the curvature. Segment density gradients can only develop in the direction perpendicular to such interface, as a mean field approximation is implemented in lattice layers 'parallel' to the interface. In the absence of density gradients, the model is equivalent to the Flory-Huggins theory. There is just one Flory-Huggins interaction parameter $(\chi)$ between monomers $\mathrm{A}$ and $\mathrm{B}$. We choose a value slightly above the critical point of the binary solvent $\left(\chi^{\text {cr }}=2 / n\right)$. Below the minimum value used for $n$ is 4 , and the interaction is chosen between $\chi=0.52-0.68$ (for more details on method and model refer supplemental material [23]).

Volume fraction profiles, $\varphi(z)$, and the lateral pressure distribution, $p(z)=-\omega(z)$, with $\omega$ as the grand potential density, are presented for the default planar tensionless $\mathrm{L} / \mathrm{L}$ interface, in Figs. (1a) and (1b). Here, $z \equiv z / b$ is the dimensionless normal coordinate. In Fig. (1a) we see that the two liquids give a Van der Waals-like profile and the accumulated copolymers have their blocks on corresponding sides of the interface. The pressure profile $p(z)$, see Fig. (1b), has a negative excursion at the interface due to the contribution from the $\mathrm{L} / \mathrm{L}$ interface and positive 'wings' on either side of the interface due to the overlap of copolymers in brush-like configuration. From earlier work [24] we know that $\gamma=-\sum_{z} p(z)$ and, that the second moment of the pressure distribution with respect to the Gibbs plane $\left(R^{g}\right)$ provides a direct estimate of $\bar{\kappa}=\sum-\left(z-R^{g}\right)^{2} p(z)$. The latter relation proved useful for the evaluating $\bar{\kappa}$ of lipid bilayers, and presents a strong test for alternative, more elaborate routes to obtain the same quantity.

Similar as for lipid bilayers, an independent alternative route for evaluating $\bar{\kappa}$ makes use of three-gradient 


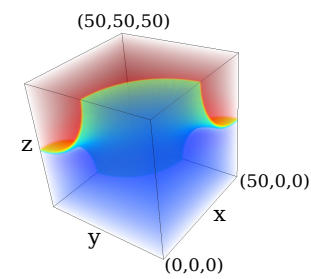

(a)

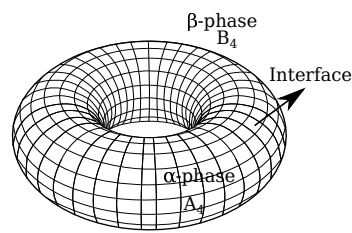

(c)

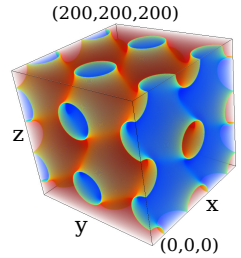

(b)

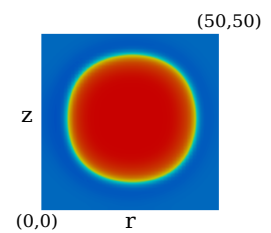

(d)
FIG. 2. Volume fraction distribution of $\alpha$-phase from 3D SCF calculation of interface modeled as Im3m cubic phase. $1 / 8$ of a unit cell is shown in (a) 8 unit cells are shown for visualization in (b). Schematic illustration of an interface in torus shape is shown in (c). Volume fraction distribution of $\alpha$-phase from 2D SCF calculation of minimal torus in a cylindrical lattice is shown in (d). The molecular model is similar as in Fig. 1. Color scale from blue to red is $0.2-0.8$ for all contours.

SCF computation as shown in Fig. (2a), where on all six faces of the elementary box, Neumann boundary conditions apply; the elementary box is $1 / 8$ th of a unit cell of an Im3m phase, 8 unit cells are shown in Fig. (2b). When equal amounts of A and B are present in the system, the interface splits the volume into two identical sub-volumes (phase $\alpha$ and $\beta$ ) while $J=0$ along the surface and $\Delta P_{L}=0$. As soon as the copolymers are added, such that the chemical potential of all molecular species is equal to the corresponding values of the planar tensionless system, we lie within an $(\mu, V, T)$-ensemble; thus, the grand potential, $\Omega=F-\sum_{j} \mu_{j} n_{j}$, is the characteristic function, and $\Omega=\bar{\kappa} \int_{M} K d A$. Using Gauss-Bonnet theorem for compact, boundary-less Riemann manifold, the integral of curvature over the area can be evaluated as $-8 \pi[25,26]$. Thus, the grand potential for the unit cell, $\Omega=-8 \pi \bar{\kappa}$. Hence, from the scale-invariant grand potential $\Omega$ directly follows $\bar{\kappa}$. The result is consistent with the second moment over the pressure profile (see Tab. (1) in supplemental material [23]).

The procedures to evaluate $\kappa$ are more involved. In Figs. (1c) and (1d), we have presented typical results for spherically and cylindrically curved interfaces when $\Delta P_{L}=0$ as a result of the adsorption of the copolymers. In Fig. (1c) we show the area per copolymer at the interface (inverse of adsorbed amount) and in Fig (1d) the corresponding chemical potentials as a function of the curvature $J$. In Fig. (1d) we notice that the chemical potentials remain constant upon bending in case of spherical curvature. This means that in this ge- ometry bending is performed in the $(\mu, V, T)$-ensemble. The reason why the system can maintain its chemical potentials upon bending of the interface is traced to the known fact that integrating Eqn. (1) over the area, $\Omega=\int_{M} \gamma(J, K) d A=4 \pi(2 \kappa+\bar{\kappa})$, is a constant irrespective of the size of the spherical droplet showing scale invariance.

Now an indirect route is available to compute $\kappa$, namely from combining the total curvature energy from the spherical droplet with the Gaussian bending modulus $\bar{\kappa}$ found above. Ideally, we would like to validate this indirect route with a direct estimate.

Again, as in the cylindrical geometry, neither the adsorbed amount of surfactant, cf. Fig. (1c), nor the corresponding chemical potential, cf. Fig. (1d), is conserved, and we cannot use this geometry to obtain $\kappa$. A direct route to evaluate the mean bending modulus is still possible using a system that features a minimal torus, as illustrated in Fig. (2c). Within SF-SCF this is realized using a two-gradient $(r, z)$ cylindrical lattice. A typical result is presented in Fig. (2d) as a density contour plot in the $(r, z)$ cross-section. From Gauss-Bonnet theorem, as the torus has genus $g=1$ the integral $\int_{M} K d A$ vanishes. Moreover, the so-called Willmore energy of the torus has contribution only from mean curvature, $W=\frac{1}{4} \int_{M} J^{2} d A$.

In 1965, T.J. Willmore conjectured that the Willmore energy $(W)$ of a smooth torus immersed in $3 \mathrm{D}$ space is always greater than or equal to $2 \pi^{2}$ [27]. This conjecture was proved by Marques and Neves in 2012 [28]. The Willmore energy reaches its minimum when the radius of revolution is $\sqrt{2}$ times the radius of the generating circle, as shown in Fig. (2d). By integrating the Helfrich equation for the toroidal configuration with minimal Willmore energy, we obtain the grand potential for torus as, $\Omega_{t}=\frac{1}{2} \kappa \int_{M} J^{2} d A=2 \kappa W=4 \pi^{2} \kappa$.

Now the protocol boils down to generating this minimal torus in SF-SCF while adding the copolymer such that $\Delta P_{L}=0$. It occurs that in this case, the system converges with all its chemical potentials equal to that of the planar tensionless interface and lies within the $(\mu, V, T)$ ensemble. Similar as in the droplet case this result is traced to the scale invariance, in this case of the minimal Willmore energy. Its grand potential gives a direct estimate of $\kappa=\Omega_{t} / 4 \pi^{2}$.

The values found for $\kappa$ by the direct route and indirect route are congruent, proving that there is complete consistency in obtaining the bending rigidities, using scaleinvariant surfaces, for tensionless balanced $\mathrm{L} / \mathrm{L}$ interfaces [23].

As we have established the molecular link for bending rigidities, we now present in Fig. (3) the chain length dependence of the bending rigidities in the regime where $N>n$. The trends support the results from simulations $[16,17,29]$. While alluring to conclude that bending rigidities have a linear dependence on the chain length of the surfactants, results in regime $n \approx N$ are contra- 

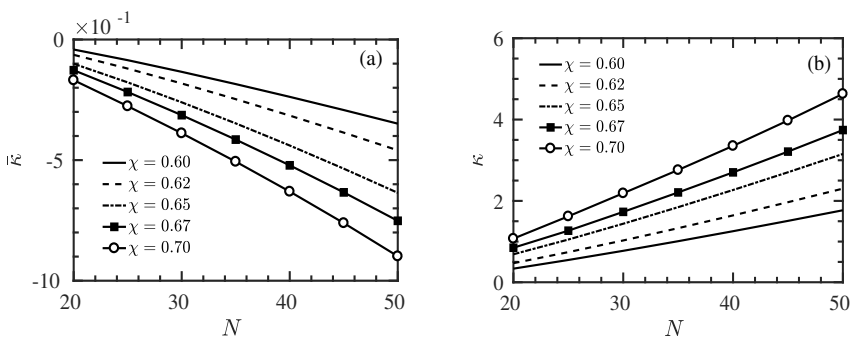

FIG. 3. Chain length dependence of bending rigidities. (a) and (b) $N>n$ Regime: Chain length of bulk phases fixed $\left(\mathrm{A}_{4}, \mathrm{~B}_{4}\right)$, surfactant chain length is varied $\left(\mathrm{A}_{N} \mathrm{~B}_{N}\right.$, where $20<\mathrm{N}<50 ; 0.6<\chi<0.7)$.

dicting this observation and do not show a similar linear dependence [see supplemental material [23] Fig. (1)]. It is observed that the surfactant chain length dependence of rigidities is strongly influenced by solvent chain length and interaction parameter between monomeric units, an important effect which has not been addressed in previous works [9-17]. A thorough analysis of the magnitude of $\kappa$ is, however, beyond the scope of the present letter and will be presented elsewhere.

Moving from the regime where the solvent length is smaller compared to the surfactant block length, $n<N$, to the regime where the solvent length is comparable to that of the surfactant, we observe that $\bar{\kappa}$ is of opposite sign (cf. Fig. (3) above and Fig. (1) of supplemental material [23]). Such a sign switch is of exceptional interest, as it addresses a topological phase transition in microemulsions to be achieved in two ways: (1) by tuning the interaction parameter for fixed solvent and surfactant lengths and (2) by tuning solvent length for fixed surfactant length and $\Delta \chi=\chi-2 / n$.

In Fig. (4a) the dependence of both $\kappa$ and $\bar{\kappa}$ are shown for surfactant block length of $N=20$ as a function of a measure of closeness to the critical point of the binary solvent $\Delta \chi$. The Gaussian bending modulus, $\bar{\kappa}$, switches from negative to positive when moved towards weak segregation; this transition occurs earlier in higher $n$ (dashed line) for fixed $N$.

A similar effect can also be achieved by tuning the surfactant chain length for a given solvents chain length and interaction energy $(\Delta \chi)$. Experimentally, one can reach weak segregation by the addition of a suitable cosolvent which diminishes the difference between the two primary solvents.

A summary of results, obtained by tuning $N$, is presented as a 'phase diagram' in Fig. (4b). The two governing parameters, i.e., block length of the copolymer and the length of the solvents are on the $x-$ and $y$-axis, respectively. The interaction parameter is chosen as $\Delta \chi$. By tuning the surfactant length for given interactions $(\Delta \chi)$ and solvent chain length, $\kappa$ increases and $\bar{\kappa}$ decreases monotonically, also showing a sign switch at the


FIG. 4. (a) Gaussian bending modulus (blue axis) $\bar{\kappa}$ [in units of $k_{B} T$ ] and mean bending modulus (red axis) $\kappa$ [in units of $\left.k_{B} T\right]$ as a function of $\Delta \chi(\Delta \chi=\chi-2 / n)$. Surfactants are modeled as $A_{N} B_{N} . \alpha$-phase is modeled as $A_{n}$ and $\beta$-phase is modeled as $B_{n}$. [solid line: $n=4$, dashed line: $n=6$ ] (b) Phase diagram in $n$ and $N$ coordinates for fixed $\Delta \chi$ as indicated. The sign and magnitude (in units of $k_{B} T$ of the rigidities are indicated.

solid lines.

These results imply the tendency of the interface to remain planar on average when $n<<N$. This result is contrasted with the situation when the length of the solvent molecules is increased to be similar, $n \approx N$, or even larger than that of the copolymer, $n>N ; \bar{\kappa}$ becomes positive in this regime while $\kappa$ is small but positive. For large $n$, we have $\bar{\kappa}>0 ; 0<\kappa<1$. These features are consistent with a sponge phase (Winsor III) [4] which grows in importance with reducing $\chi$. Stable but very flexible, and strongly fluctuating lamellar phases (as $\bar{\kappa}<0$ and $0<\kappa<1$ ) are observed as $N$ is increased for fixed $n$, whereas for very large $N, \kappa$ is $>1$, and we enter a region where the fluctuations of the interface are weak, crossing the dashed lines, as shown in Fig. (4b).

We have linked molecular characteristics to bending rigidities for surfactant-covered $\mathrm{L} / \mathrm{L}$ interfaces. Using surfaces with scale-invariant curvature energies is embellished as an elegant route to determine $\kappa$ and $\bar{\kappa}$ unambiguously; this route cautiously exploits the tensionless state of the interfaces and avoids the linear term in curvature. Large deviations from these constraints imply the loss of the microemulsion middle-phase in favor of emulsions with oil-in-water or water-in-oil droplets; however, understanding the effects of small deviations is vital, as it is a prerequisite for any detailed comparison with experiments. The current analysis provides a natural starting/reference point to generalize for molecular asymmetry, spontaneous curvature and finite tension of the interfaces.

This work is part of an Industrial Partnership Programme, 'Shell/NWO Computational Sciences for Energy Research (CSER-16)', of the Foundation for Fundamental Research on Matter (FOM), which is part of the Netherlands Organisation for Scientific Research (NWO). Project number: 15CSER26. We are indebted to T. E. Kodger for helpful discussions. 
* Author to whom correspondence should be addressed: R. Varadharajan: ramanathan.varadharajan@wur.nl

† F.A.M Leermakers: frans.leermakers@wur.nl

[1] P. G. de Gennes and C. Taupin, J. Phys. Chem. 86, 2294 (1982).

[2] C. Tanford, The Hydrophobic Effect: Formation of Micelles and Biological Membranes (J. Wiley., 1980).

[3] S. Schacht, Q. Huo, I. G. Voigt-Martin, G. D. Stucky, and F. Schuth, Science 273, 768 (1996).

[4] G. Gompper, M. Schick, and S. Milner, "Self-assembling amphiphilic systems," (1995).

[5] S. A. Safran, Statistical thermodynamics of surfaces, interfaces, and membranes, Vol. 90 (Perseus Books, 1994).

[6] S. Safran, D. Roux, M. Cates, and D. Andelman, Physical review letters 57, 491 (1986).

[7] L. Scriven, Nature 263, 123 (1976).

[8] M. Cates, D. Roux, D. Andelman, S. Milner, and S. Safran, EPL (Europhysics Letters) 5, 733 (1988).

[9] T. Hellweg and D. Langevin, Phys. Rev. E 57, 6825 (1998).

[10] E. Kurtisovski, N. Taulier, R. Ober, M. Waks, and W. Urbach, Phys. Rev. Lett. 98, 258103 (2007).

[11] I. Szleifer, D. Kramer, A. Ben-Shaul, D. Roux, and W. M. Gelbart, Physical review letters 60, 1966 (1988).

[12] A. Würger, Phys. Rev. Lett. 85, 337 (2000).

[13] M. Gradzielski, D. Langevin, and B. Farago, Physical Review E 53, 3900 (1996).

[14] M. Gradzielski, D. Langevin, T. Sottmann, and R. Strey, The Journal of chemical physics 106, 8232 (1997).
[15] C. R. Safinya, E. B. Sirota, D. Roux, and G. S. Smith, Phys. Rev. Lett. 62, 1134 (1989).

[16] L. Rekvig, B. Hafskjold, and B. Smit, Phys. Rev. Lett. 92, 116101 (2004).

[17] F. M. Thakkar, P. K. Maiti, V. Kumaran, and K. G. Ayappa, Soft Matter 7, 3963 (2011).

[18] F. A. M. Leermakers, The Journal of chemical physics 138, 04B610 (2013).

[19] W. Helfrich, J. Charvolin, J. F. Joanny, and J. ZinnJustin, Les Houches Session XLVIII (1988) (1990).

[20] J. M. H. M. Scheutjens and G. J. Fleer, J. phys. Chem 83, 1619 (1979).

[21] G. Fleer, M. A. C. Stuart, J. M. H. M. Scheutjens, T. Cosgrove, and B. Vincent, Polymers at interfaces (Springer Science \& Business Media, 1993).

[22] R. A. Kik, F. A. M. Leermakers, and J. M. Kleijn, Physical Review E 81, 021915 (2010).

[23] See Supplemental Material http://link.aps.org/ for details about method and additional figures..

[24] S. M. Oversteegen and F. A. M. Leermakers, Physical Review E 62, 8453 (2000).

[25] S. S. Chern, Annals of mathematics , 747 (1944).

[26] W. Fenchel, Journal of the London Mathematical Society 1, 15 (1940).

[27] T. J. Willmore, An. Sti. Univ. "Al. I. Cuza" Iasi Sect. I a Mat.(NS) B 11, 493 (1965).

[28] F. C. Marques and A. Neves, Annals of Mathematics 179, 683 (2014).

[29] B. Smit, P. A. J. Hilbers, K. Esselink, L. A. M. Rupert, N. M. Van Os, and A. G. Schlijper, Nature 348, 624 (1990). 